\title{
Research on the Railroad Bridge Monitoring Platform Based on the Internet of Things
}

\author{
Ying $\operatorname{Sun}^{1}$ \\ ${ }^{1}$ Department of Electronic and Information Engineering, \\ LiaoningRailway Vocational and Technical College, 121000 \\ sunyingjz@sina.com
}

\begin{abstract}
As railroad bridge is one of the most important infrastructures of railway, the safety of bridge has a direct effect on railway operation efficiency and safety. In this pdper, we propose a distributed railway bridge monitoring platform based on the Internet of Things (IOT) to monitoring the state of bridge safety. We made detalled research on the sensor nodes deployment, sensing data updating and early warning mechanisms of the wireless sensor network. The proposed system achieved the desired purpose of reat-time monitoring of railroad bridge safety during test in China Railwas Gorp.

Keywords: railroad bridge safety; Internet of Things, Wikeless sensor network; monitoring platform

\section{Introduction}

As an important infrastructure of the country, as the artery of national economy and as a popular traffic tool, railway plays an impgrtant role in modern logistics system. As one of the important infrastructure of railway, railroad bridge directly affect the safety of railway traffic status. So the research on the safety of railroad bridges has important practical significance [1]. Railway bridge has the charaeteristics of large load, large impact force, traffic density, high requirements of the standard for resistance to natural disaster. Therefore, periodic rapid detection of infrastructure $1 \mathrm{~s}$ a necessary measure to master the railway bridge safety condition and ensure the afety.

At present, there are many long-span bridges have deployed large health monitoring systems, mainly for real-time monitoring of vibration response, strain and deformation of structural mechanics, thus to evaluate the operation of the safety status of the bridge. There are a lot of traditional methods for bridge monitoring such as the low strain reflected wave method, the sound wave transmission method, the core method and so on. The existing methods of bridge safety monitoring has many limitations and inconvenience such as the incomplete judgment of multiple defects, the inaccurate judgment of the nature of the defect, the long time and high cost of monitoring, which has a risk of false positives or false negatives .

Compared with the traditional measurement methods, satellite positioning of the bridge monitoring method can not only achieve the $3 \mathrm{D}$ high precision measurement of a day of uninterrupted, but also has the characteristics of large range, short detention, strong real-time and synchronous measurement. However, the above researches and tests are all for the road bridges and none for railway bridges [2]. With the development of China's high-speed 
railway, higher requirements are put forward on security of railway bridges, so the study of railway bridge monitoring is imperative.

The emerging technology of wireless sensor network (WSN), which consists of a large number of sensing nodes each capable of sensing, processing and transmitting environmental information, has the advantages of sensor nodes highly integrated, low cost, low power consumption, network flexibility and so on. As a new technology of the IOT, WSN has broad application prospects in environmental monitoring. Using wireless sensor network for monitoring of railway bridges can help to reduce costs, increase flexibility and intelligent. This paper proposed a railroad bridge monitoring platform based on the Internet of Things to monitoring the operation state of the bridge, and we performed detailed study of sensor deployment, upload and and warning mechanism thus to ensure design rationality.

The structure of the paper is as follows: in this section, we give the background of the ${ }^{\circ}$ Security research status of railway bridges and review of our proposed monitoring platform. Section 2 describes the system framework. The main discussing of this paper is described in section 3-5, which includes the deployment of sensor nodes, data collection and uploading, and early warning data processing. Section 6 provides a summaty and some future work of the paper.

\section{System Framework}

The railroad monitoring platform system designed in this paper can be divided into two parts, namely are outfield and monitoring center. The outfield refers to railroad bridges and railway line where we deployed sensors to collect data, targeted and rational sensor deployment strategy are used to collec sensing data of different types of sensors; we use different ways of data upfoading for emergency data and daily data. The monitoring center consists of the monitoring sever and database, which is used to perform centralized data processing thus to achieve real-time monitoring of the railway bridge safety sate.

The proposed monitoring system uses a hybrid deployment of multimedia sensor nodes and ordinary sensor nodes. The specific/deploy method will be introduced in the Section 3. It can communicate with each other beween nodes of one type or the two nodes of different types. It use Ad Hoc network to build a network, multimedia nodes are used to collect image information such as bridge craeks, and ordinary nodes are used to sense data such as pressure of bridge deck, soil temperatare, and piers displacement information. After data compression, encryption and fusion, the data are sent to the sink node through multi-hop communication, and finally sent to the monitoring host via train or internet depending on the urgency of different data. The specific transmission and upload process will be described in Section 4. The monitoring center is to receiving, storage, analysis, process and display the collected data, users can perform monitoring, querying and processing the entire bridge information by handling the-monitor host. The monitoring platform also has early warning mechanism and security level assessment mechanism to ensure the safe use of the bridge. The specific early warning mechanism will be introduced in the Section 5. System architecture of the entire railroad monitoring platform is shown in Figure 1. 


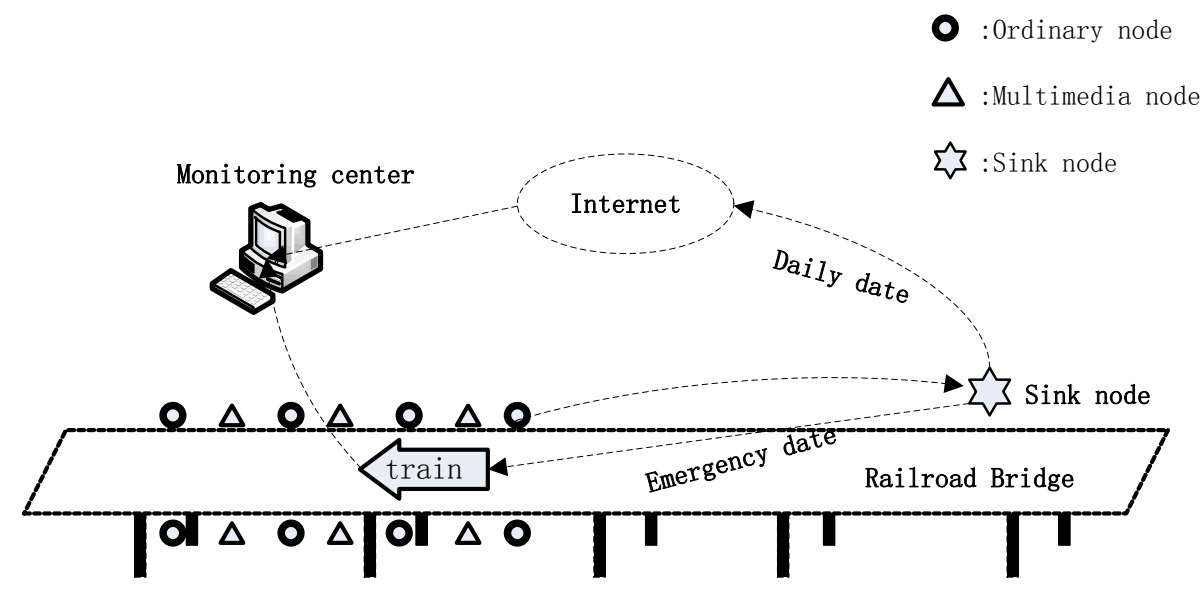

Figure 1. Framework of monitoring system

\section{The Deployment of the Wireless Sensor Network}

As the basis of the Railway bridge monitoring platform, wireless sensor network provide bridge monitoring information collection, pre-processing and transmission for the Railway bridge monitoring platform. Whether the topology of the vireless sensor network is reasonable or not will make a great impact on the performance of the whole network. In this paper, the sensor deployment of the wirelesssensor network is divided into two parts: the sensor node deployed on the bridge and the sensor node deployed around railway line.

\subsection{The sensor node deployed on the bridge}

The main task of the sensor nodes deployed on the bridge is to monitor the security state of the bridge. The sensor nodes include pressure sensors, humidity sensors, temperature sensors, deformation sensors and even wireless multimedia sensors. They can give a multi-level and comprehensive monitor of the bridge.

Pressure sensors are mainly deployed on the pier and the underside of the bridge deck bracket, especially the major veight-bearing position. They are used to real-time monitor whether the pressure has enormouls changes. The sudden change of the pressure implies that the bridge structure is undergoing a certain deformation. Usually, the position where the pressure becomes larger is more prone to damage.

Humidity sensors are mainly deployed on the bridge near the water or the culvert. When the humidity is too high, the bridge surface and the internal steel stent are prone to corrosion, results in a great damage to the bridge. Railway safety department should monitor the humidity changes of the key position of the bridge timely. And then give a maintenance and drainage timely.

The temperature of the soil surrounding the bridge has a great impact of the bridge security, especially when the temperature is too low, this easily leads to the change of the soil and results in the change of the whole position of the bridge. In extreme cases, the entire bridge will has a deviation. Hence, temperature sensors are mainly deployed on the lower bridge and the inside of the surrounding soil to monitor the change of the soil in extreme weather.

Deformation sensors are deployed on the road surface of the bridge where is more likely to have a greater deformation. The deformation of the bridge road surface usually implies the change of the bridge support structure. Railway safety department should combine with 
pressure sensors and other sensors to give an overall judgment of the security state of the bridge.

Bridge bearing is an important part of the bridge structure, to which the shift can easily occur by the influence of concrete shrinkage, temperature changes, and the traffic load. The displacement sensor can implement the real-time detection of bridge bearing shift, which should be combined with pressure sensors and other sensors' data for the integrity judgment of bridge safety.

Wireless multimedia sensor is a new type of sensor which has the function of collecting and processing multimedia information such as audio and image information. In the bridge monitor network, we mainly use the wireless image sensor. Image sensor is a positive complement for other types of sensors. It plays an supervisory role on malicious behavior against the bridge safety.

\subsection{The sensor node deployed around railway line}

According to the features of the railway, [3] designed node deployment scenarios and it achieved the goals of energy saving and extending the network lifetime. In this paper, the main task of the sensor node deployed around railway lines is to deliver the data monitored by the bridge sensors safely and quickly to the nearest moritor center se that the monitor center can have a real-time control of the bridge security. Because of the simplicity of its application, this paper intends to use the homogeneous communication node. There is no difference between sensors on the communication range, energy and other aspects. Besides, considering the communication tasks of the sensors deployed on every position are the same, this paper deploys the sensor symmetrical on the both side of the railway line, for the purpose of load balancing and network life extepding

\section{Information Collection based Wireless Sensor Nodes}

Our monitoring platform,system of railway bridges based Internet of Things is divided into two parts: outfield node information acquisition and monitoring center. Information collecting is realized by deploying corresponding sensors on the bridge or around the railway line depending on the monitoring requirements. Sensor bridge monitoring platform mainly detects the two diseases about railway fridges: bridge support displacement detection and bridge crack detection. Bridge suppot tisplacement detection is achieved by displacement sensors [4], and bridge crack detection is achieved by multimedia sensor nodes which can collect, process, transfer crack picture and other multimedia information.

The original monitoring data real-time collected by sensor node can be divided by the local threshold determination to two types: the emergency data and daily data. The emergency data is the bridge real-tine monitoring data that indicates a serious threat to the bridge's safety, for example that the displacement measured by the crack displacement sensors or the crack size measure by nultimedia sensor is over a pre-set threshold, which is the emergency data; daily data is the data which do not indicate a threat to the security of the bridge. The transmission is different for urgent data and daily data.

\subsection{Emergency data transmission and uploading}

Because the emergency data indicates a serious threat to the bridge's safety, so it is necessary to send back the data to the monitoring center for threat verdiction. External sensor monitoring network formed by the sensor nodes sends the emergency data directly to the monitoring center via the Internet. Monitoring center parses and processes the emergency data, and transfers the collected data to the displacement of bridge support or crack decision 
level. When the result of verdiction is beyond the predefined normal range, the alarm will automatically alarm, so as to truly achieve the remote real-time monitoring and improve the efficiency of support inspection maintenance and replacement maintenance.

\subsection{Daily data transmission}

In order to reduce the redundant information of sensor in space and time, so that to reduce unnecessary data transmission thus to save sensor network node energy consumption, the daily data that is not urgent to process, will be process for data fusion before transmitted to the monitoring center. Data fusion process is shown in Figure 2. The sensor nodes are clustered based on different sensor types. The sensor nodes in the same cluster monitor the same type of data information. There is a certain degree of information redundancy because of the close distance between the nodes [5]. Each node uploads its own perception data to the cluster head nodes, cluster head nodes upload the fusion data by local computing to the sink nodes, then the sink node transmits the final fusion results to the monitoring center/database. The database with the accumulation of the essential data in a long time, can accurately reflect the working state of the monitored railway bridge, and timely find development tendency for diseases of bridge body and bridge support, which enable to provide dedision basis for inspection maintenance especially jack-up replacement and ogher plank

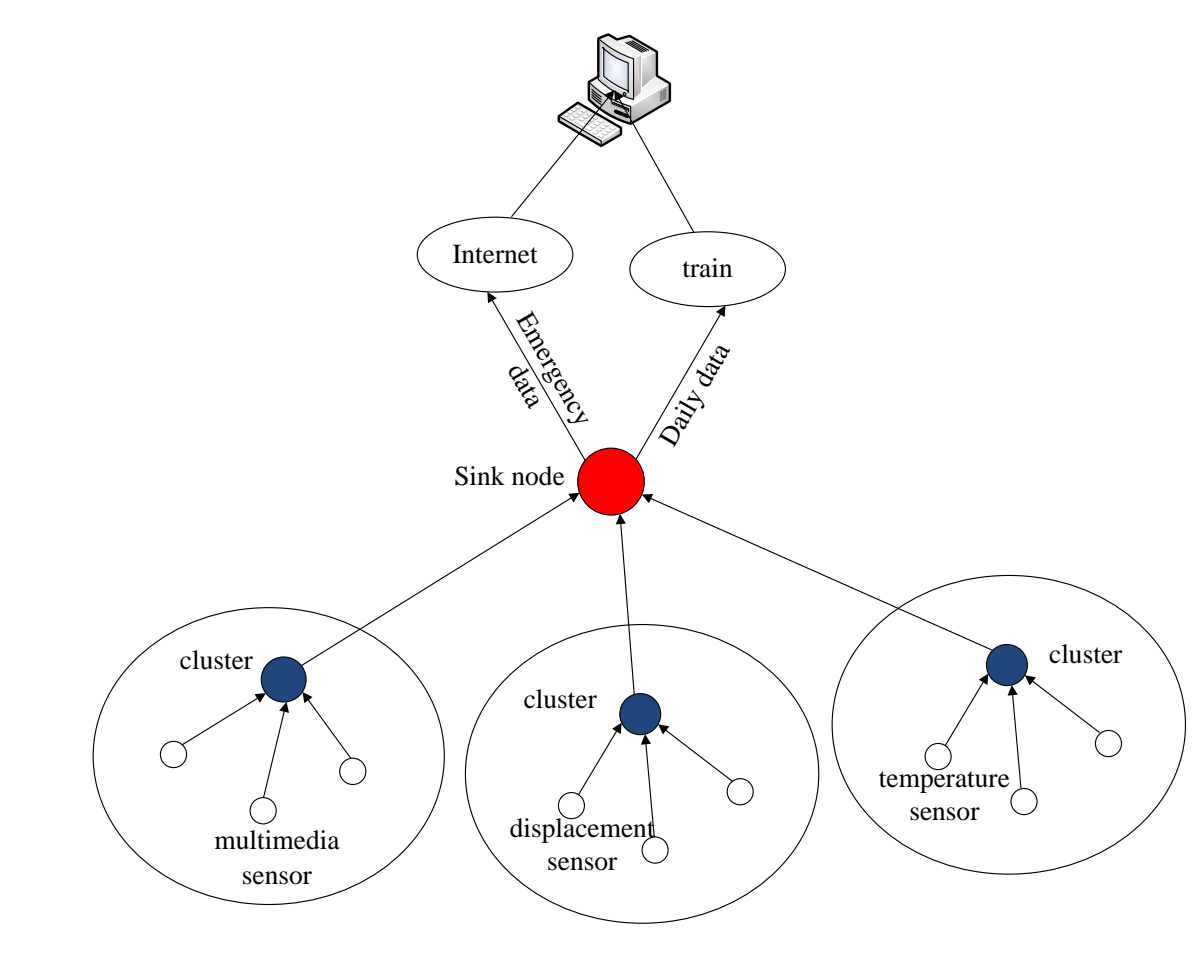

Figure 2. Data fusion process

\section{Early Warning Data Processing}

After the monitoring center collected the sensing data uploaded by the sink node, a certain pretreatment process will be preceded to gain the current safety status grade of the bridge. Security level of the bridge should be set according to the actual demand. For example, it can be classified as normal, yellow warning, orange early-warning and red warning, and each security level has a corresponding threshold. If the fusion results exceeded the preset 
threshold, the alarm monitoring system shall raise the alarm and inform the appropriate warning levels promptly. When maintenance personnel received warning, hazard would be identified and appropriate measures would be taken to eliminate the causes of the threat.

\subsection{Repeatedly warning mechanism}

In the practical application of bridge safety monitoring wireless sensor networks, sensor malfunction is an inevitable phenomenon. The accuracy of sensing data measured by sensors can directly affect decision of the system. In extreme circumstances, some sensors are completely broken will cause mistakes in the operating condition assessment of bridge, which may even lead to security incidents [6]. Sensor malfunction modes are mainly biased failure, shock failure, short circuit fault, open circuit failure and drift failure [7]. Monitoring wireless sensor network consists of various types of sensors, such as displacement sensors, temperature sensors and multimedia sensors. In the actual bridge safety monitoring network, the number of these sensors is often great. When the transmitting data fluctuation of a sensor network node is large, the monitoring system may cause an error level of security assessments, and thus gives an incorrect warning message.

To avoid early warning errors information misleading regulators, repentedly warning approach should be taken to determine the correctness of the warning message. After early warning monitoring system informed, measures need not be taken immedhate to deal with the problem. But can wait for a certain time, to observe hether the data received warning again. If the warning data consecutive time exceeds aøpredetermined value, a security problem could be determined. And the problem should be solyed by human intervention. The way that repeatedly alert would largely avoid the occurrence of wrong positives. In addition, there're other means to provide system stability and accurac for example employing sensors have superiority in stable performance or adopting accurate fault detecting techniques [8].

With growing of using time, bridges and other infrastructure will be gradually maturing, and the security situation will be gradually reduced. Obviously, we should not use the security level for new bridge to assess that of an old bridge. Therefore, the appropriate threshold should be set for bridges of different using time to evaluate the security level of different bridges. For example, the crack on the bridge is an important indicator to determine the safety level of a bridge [9]. Monitoring indicators of bridge cracks include length, width, and the crack growth rate and so on. For hewly built bridge, assessments standard tend to be very strict, even a quite short crack is not allowed. While assessing a bridge built 20 years ago, the standard willbe lowered afot for the old bridge cracks are inevitable.

\subsection{Automatically threshold adjusting based on the feedback learning}

Referencing dertain standards, security threshold level can be manually set according to the actual situation. However, many practical situations complicated than imagined, artificial setting is often not accurate. If a dynamic threshold adjustment algorithm can be built, the current security level of bridge will be automatically assessed according to the actual situation of the bridge, and this algorithm should have robustness and adaptability. The neural network algorithm is a good way to solve this problem.

Neural network algorithm has self-learning ability, and is widely used in pattern recognition, optimization, data compression, and other areas [10].After inputting certain priori data, artificial neural networks using some learning algorithms can automatically determine the weight and give an optimal classification results. Figure 3 illustrated the automatically threshold adjusting processing used in this paper. 
Figure 3 illustrates the threshold level automatic adjustment process based on feedback learning mechanism (in this case, the "feedback" is not the error back propagation (BPF) neural network). When setting an initial threshold, enough sample data should be input to ensure the neural network adjust the network weights according to the setting of the initial threshold automatically and achieve enough accuracy. In the late process of monitoring, as time goes on, the bridge monitoring system will compute and input the collected data to the neural network repeatedly, and then the final evaluation results will be achieved. If the result is normal, the results will be stored as the training data. After normal monitoring data is sufficient, the recent input data (for example, 100) will be the training data to train the neural network. With the circulation the neural network weights will change gradually, which is suitable for bridge safety status. As the training process is relatively slow, even there is an error during the process, the neural network can still give an accurate judgment and send ${ }^{\circ}$ warning information.

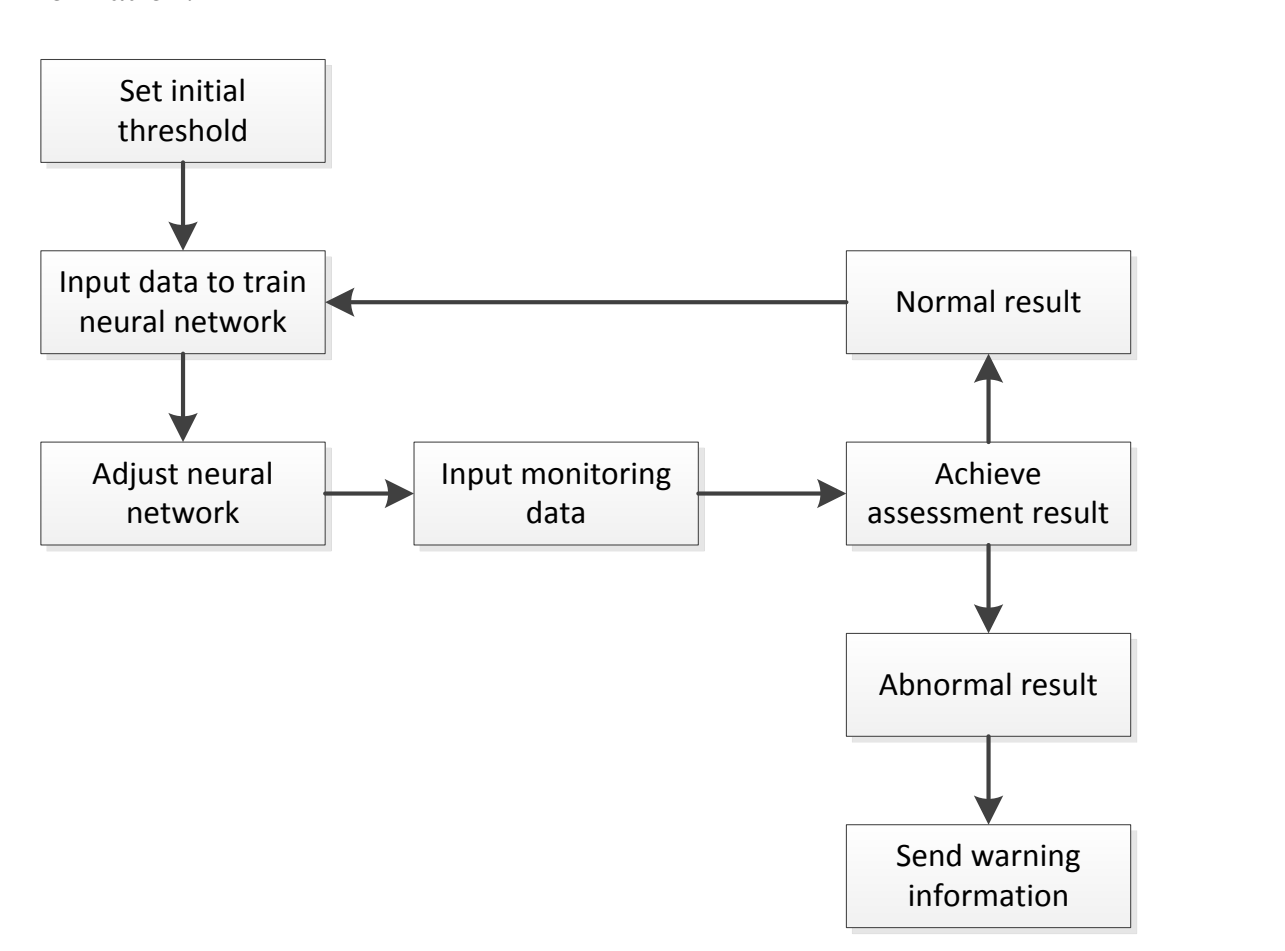

Figure 3. A threshoid automatic adjustment process based on feedback

\section{Conclusion}

The railway bridge monitoring platform based on the Internet of Things proposed in this paper mainly includes three key modules: wireless sensor's deployment, wireless sensor nodes' information collection and warning information's processing. During the test period in China Railway Corp, the platform's performance is excellent, for it can detect various kinds of common bridge damage accurately, which is significant for the safety of the railway bridge. As the next step of our work, we will set up a complete set of railway safety monitoring system, which includes the real-time safety monitoring of infrastructures along the railway such as the railway station, bridge, tunnel, line and so on. It will provide the railway system a full range of security early warning, not only confined to the railway bridge any more. 


\section{References}

[1] J. Zheng, "Railway Passenger Transportation Development in China", Urban Transport of China, vol. 8, no. 1, (2010) January.

[2] T. W. Hou, S. Guo and T. L. Han, "Simulations of safety monitoring for single railway bridge", Sensor World, vol. 18 , no. $3, \mathbf{( 2 0 1 2 )}$.

[3] J. S. Fu, Z. J. Zhang and Y. Liu, "Research and Simulation on An New Algorithm RTST for Energy-Efficient Coverage in URail Traffic Wireless Sensor Networks", Journal of the China Railway Society, (2013).

[4] Z. H. Xia, S. F. Zhu and Z. H. Zong, "Assessment of support displacement of a continuous rigid-frame bridge with a large span based on long-term health monitoring", Proceedings in 18th National Conference on Structural Engineering, (2009), GuangZhou, China, pp. 542-548.

[5] R. Wu, "Research on Data Fusion Technology of Wireless Multimedia Sensor Networks", Nanjing University of Posts and Telecommunication, (2013).

[6] T. H. Guo and J. Nurre, "Sensor failure detection and recovery by neural networks", Neural Networks, IJCNN-91-Seattle International Joint Conference on IEEE, vol. 1, (1991), pp. 221-226.

[7] S. K. Yung and D. W. Clarke, "Local sensor validation", Measurement and Control, (1989).

[8] M. Henry, "Sensor validation and fieldbus", Computing \& Control Engineering Journal, vol. 6, no. 6, (1995), pp. 263-269.

[9] I. Abdel-Qader, O. Abudayyeh and M. E. Kelly, "Analysis of edge-detection techniques for crack identification in bridges", Journal of Computing in Civil Engineering, yol. 17, no. 4, (2003), pp. 255-263.

[10] C. Baladrón, J. M. Aguiar and L. Calavia, "Performance study of the application of artificial neural networks to the completion and prediction of data retrieved by underwate sensors", Sensors, vol.12, no. 2, (2012), pp. 1468-1481.

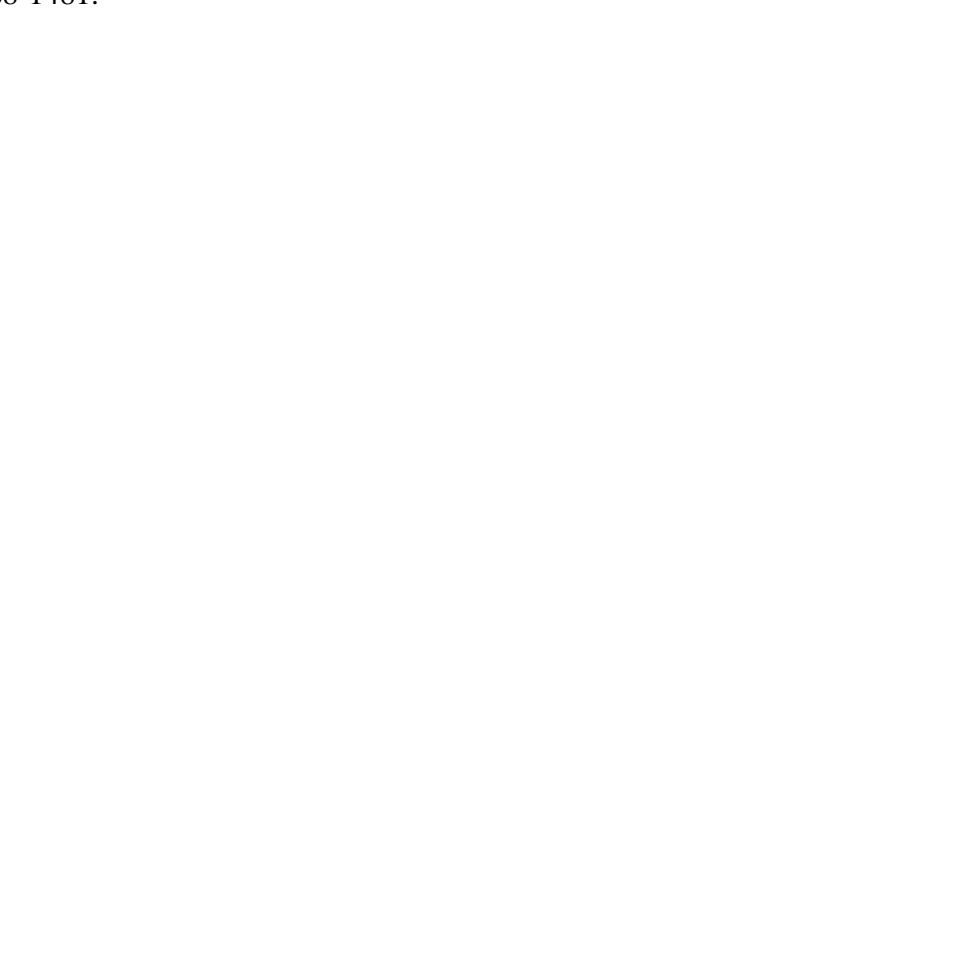

